\title{
Correlation between the Stability of Substituted Cobaltocenium and Molecular Descriptors
}

Shehani T. Wetthasinghe, ${ }^{\dagger}$ Chunyan Li, ${ }^{\ddagger}$ Huina Lin, ${ }^{\dagger}$ Tianyu Zhu, ${ }^{\dagger}$ Chuanbing Tang, ${ }^{\dagger}$ Vitaly Rassolov, ${ }^{\dagger}$ Qi Wang, ${ }^{*, \dagger}$ and Sophya Garashchuk*, ${ }^{*}$

$\dagger$ Department of Chemistry and Biochemistry, University of South Carolina, Columbia, SC

$\ddagger$ Department of Mathematics, University of South Carolina, Columbia, SC

E-mail: qwang@mailbox.sc.edu; garashchuk@sc.edu 
Fig. S1. Substituent groups considered in modification of $\mathrm{CoCp}_{2}^{+}$and $\mathrm{RhCp}_{2}^{+} \quad$ p. S3

Fig. S2. Bond dissociation energy (BDE) for methyl substituted cobaltoceniums p. S4

Table S1. Spin-multiplicities and energies of $\mathrm{CoCp}_{2}^{+}$and its fragments p. S5

Table S2. Dissociation energies of $\mathrm{CoCp}_{2}^{+}$and its fragments p. S5

Table S3. Dataset for the mono-substituted $\mathrm{CoCp}_{2} \mathrm{OH} \quad$ p. S6

Table S4. Dataset for the di-substituted $\mathrm{CoCp}_{2} \mathrm{OH} \quad$ p. S7

Table S5. Dataset for the hetero-substituted $\mathrm{CoCp}_{2} \mathrm{OH} \quad$ p. S8

Table S6. $\mathrm{CoCp}_{2}^{+} \mathrm{OH}^{-}$derivatives excluded from the LSF and CIFNN analysis p. S9

Table S7. Dataset for the di-substituted $\mathrm{RhCp}_{2}^{+} \mathrm{OH}^{-} \quad$ p. S10

Table S8. Optimal coefficients of the LSF models using $H$-charge on $\mathrm{C}_{6} \mathrm{H}_{5} \quad$ p. S11

Table S9. Optimal coefficients of the LSF models using $H$-charge on $\mathrm{C}_{5} \mathrm{H}_{4} \quad$ p. S11

Table S10. Hyperparameters and number of parameters of the CIFNN models p. S11 


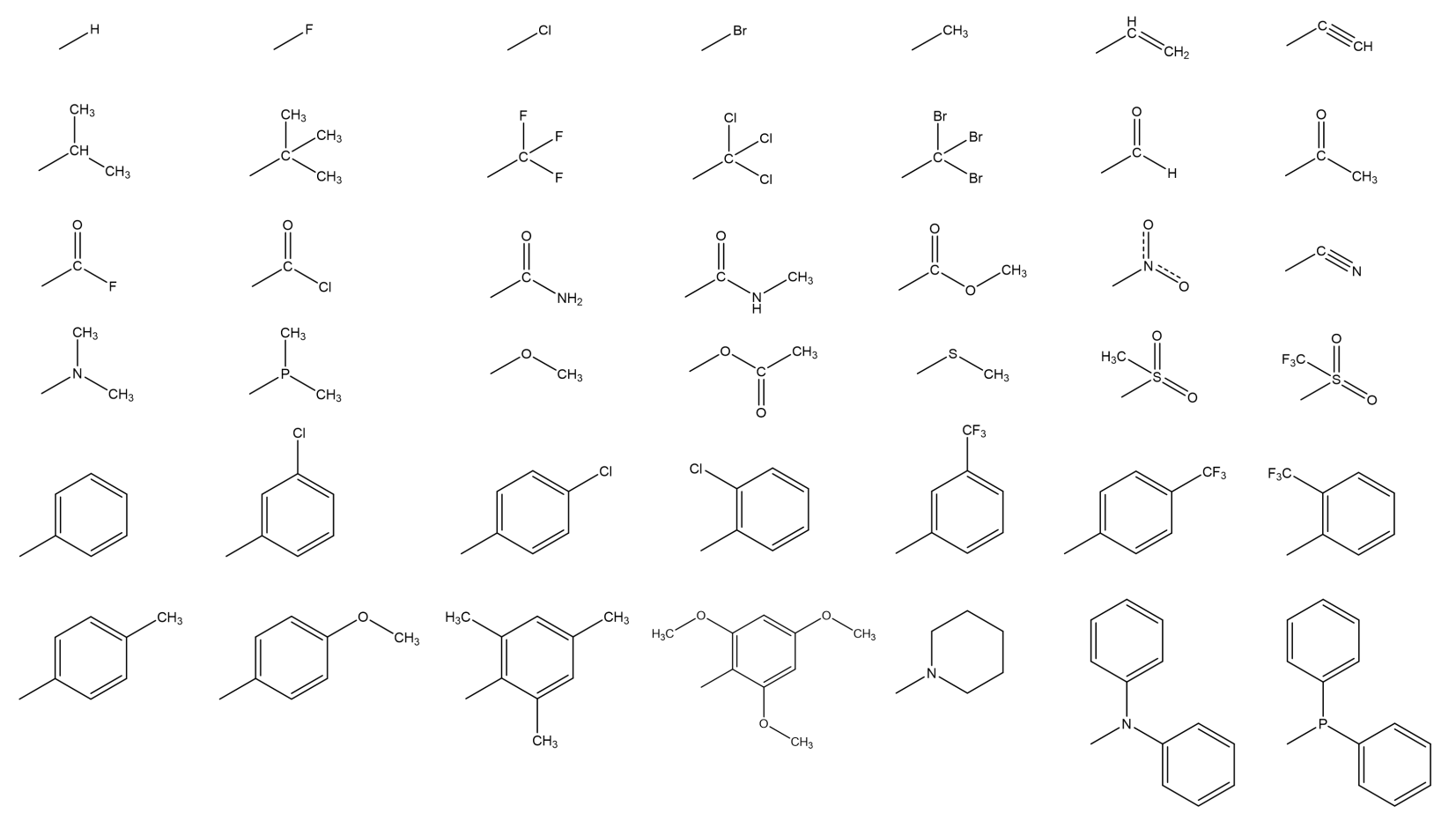

Figure S1: The substituent groups, $\mathrm{Y}$, considered in modification of $\mathrm{CoCp}_{2}^{+}$and $\mathrm{RhCp}_{2}^{+}$. 
a)

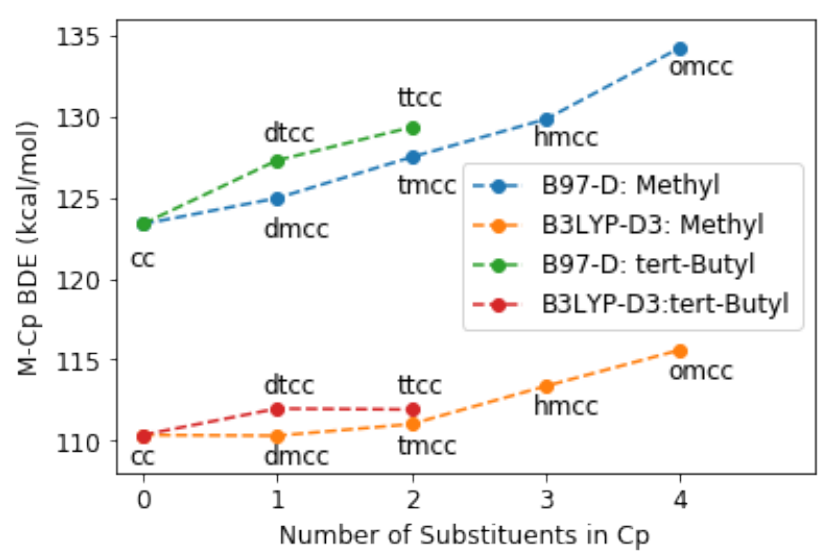

b)
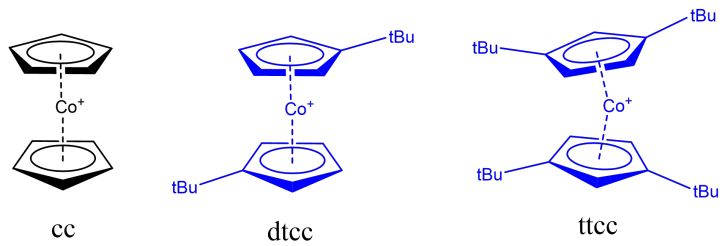

ttcc

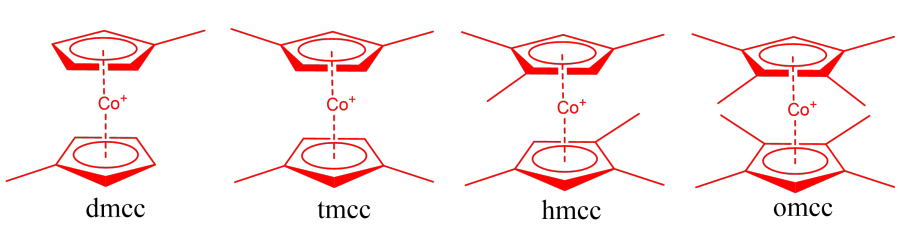

Figure S2: (a) The bond dissociation energy (BDE) for the methyl and tert-butyl substituted cobaltocenium species shown in panel (b). The BDEs were computed in gas phase using B97D/(def2,def2-SVP) method employed in Ref. ${ }^{1}$ and B3LYP-D3/(m6-31G,6-31G**) method used in this work. The experimental value for the unsubstituted $\mathrm{CoCp}_{2}^{+}$is $105.4 \pm 3.5$ kcal/mol. ${ }^{2}$ 
Table S1: Dissociation energy of $\mathrm{CoCp}_{2}^{+} \mathrm{OH}^{-}$complex in gas and aqueous phases computed at B3LYP-D3/(m6-31G*,6-31G**) level (m6-31G* is used for Co). Aqueous phase is represented by the Polarized Continuum Model (PCM). $\Delta$ is the difference between the energy of the fragments and that of the complex for the respective channel.

\begin{tabular}{|l|c|c|c|c|}
\hline phase & \multicolumn{2}{|c|}{ gas } & \multicolumn{2}{c|}{ aqueous } \\
\hline system & $\mathrm{E}\left[\mathrm{E}_{h}\right]$ & $\Delta\left[\frac{\mathrm{kcal}}{\text { mol }}\right]$ & $\mathrm{E}\left[E_{h}\right]$ & $\Delta\left[\frac{\mathrm{kcal}}{\text { mol }}\right]$ \\
\hline $\mathrm{CoCp}_{2}^{+} \mathrm{OH}^{-}$ & -1845.487 & 0 & -1845.521 & 0 \\
$\mathrm{Co}^{2+} \mathrm{CpOH}^{-}+\mathrm{Cp}^{-}$ & -1845.223 & 165.6 & -1845.422 & 62.1 \\
$\mathrm{Co}^{+} \mathrm{CpOH}^{-}+\mathrm{Cp}$ & -1845.457 & 18.7 & -1845.457 & 26.6 \\
\hline
\end{tabular}

Table S2: Energies of $\mathrm{CoCp}_{2}^{+}$and its fragments within PCM for different spin states. The electronic structure methods employed are DFT based on B3LYP-D3 functional and MP2 theory paired with $\mathrm{m6}-31 \mathrm{G}^{*}$ basis set for $\mathrm{Co}$ and $6-31 \mathrm{G}^{* *}$ basis sets for $\mathrm{C}, \mathrm{H}$ and $\mathrm{O}$ atoms. The lowest energy for each species is printed in bold.

\begin{tabular}{|c|c|c|c|c|c|}
\hline \hline & multiplicity & \multicolumn{2}{|c|}{$\mathrm{S}^{2}$} & \multicolumn{2}{c|}{ energy $\left[\mathrm{E}_{h}\right]$} \\
\hline \multirow{2}{*}{ system } & $2 \mathrm{~S}+1$ & $\mathrm{~B} 3 \mathrm{LYP}$ & $\mathrm{MP} 2$ & $\mathrm{~B} 3 \mathrm{LYP}$ & $\mathrm{MP} 2$ \\
\hline \multirow{2}{*}{$\mathrm{CoCp}_{2}^{+}$} & 1 & 0.00 & 0.00 & $\mathbf{- 1 7 6 9 . 5 7}$ & $\mathbf{- 1 7 6 7 . 0 8}$ \\
\cline { 2 - 6 } & 3 & 2.06 & 2.12 & $\mathbf{- 1 7 6 9 . 4 9}$ & $\mathbf{- 1 7 6 6 . 8 8}$ \\
\hline \multirow{2}{*}{$\mathrm{CoCp}^{+}$} & 2 & 1.39 & 0.78 & $\mathbf{- 1 5 7 5 . 8 8}$ & -1573.92 \\
\cline { 2 - 6 } & 4 & 3.76 & 3.77 & $\mathbf{- 1 5 7 5 . 9 0}$ & $\mathbf{- 1 5 7 3 . 9 8}$ \\
\hline \multirow{2}{*}{$\mathrm{Cp}$} & 2 & 0.77 & 1.01 & $\mathbf{- 1 9 3 . 4 7}$ & $\mathbf{- 1 9 2 . 8 2}$ \\
\cline { 2 - 6 } & 4 & 3.76 & 3.77 & $\mathbf{- 1 9 3 . 3 4}$ & $\mathbf{- 1 9 2 . 6 9}$ \\
\hline
\end{tabular}


Table S3: Dataset for the mono-substituted $\mathrm{CoCp}_{2}^{+} \mathrm{OH}^{-}$; B3LYP-D3/(m6-31G*,6-31G**), aqueous PCM.

\begin{tabular}{|c|c|c|c|c|c|c|c|c|c|}
\hline $\begin{array}{c}\text { substituent } \\
\mathrm{Y}\end{array}$ & $\begin{array}{l}\mathrm{BDE} \\
{\left[\frac{k c a l}{m o l}\right]}\end{array}$ & $\begin{array}{c}\mathrm{E}_{\mathrm{HOMO}}^{(1)} \\
{\left[\mathrm{E}_{h}\right]}\end{array}$ & $\begin{array}{c}\mathrm{E}_{\mathrm{LUMO}}^{(1)} \\
{\left[\mathrm{E}_{h}\right]}\end{array}$ & $\begin{array}{c}\mathrm{E}_{\mathrm{HOMO}}^{(2)} \\
{\left[\mathrm{E}_{h}\right]}\end{array}$ & $\begin{array}{c}\mathrm{E}_{\mathrm{LUMO}}^{(2)} \\
{\left[\mathrm{E}_{h}\right]}\end{array}$ & $\begin{array}{c}\mathrm{E}_{\mathrm{HOMO}}^{(3)} \\
{\left[\mathrm{E}_{h}\right]}\end{array}$ & $\begin{array}{c}\mathrm{E}_{\mathrm{LUMO}}^{(3)} \\
{\left[\mathrm{E}_{h}\right]}\end{array}$ & $\begin{array}{c}\mathrm{H}_{C_{6} H_{5}}^{(3)} \\
{[\mathrm{e}]}\end{array}$ & $\begin{array}{c}\mathrm{H}_{C_{5} H_{4}}^{(3)} \\
{[\mathrm{e}]}\end{array}$ \\
\hline$-\mathrm{N}\left(\mathrm{CH}_{3}\right)_{2}$ & 15.718 & -0.1864 & -0.0628 & -0.2131 & -0.0789 & -0.1594 & -0.0809 & 0.0118 & -0.1831 \\
\hline$-\mathrm{OCH}_{3}$ & 15.239 & -0.1858 & -0.0712 & -0.2131 & -0.0789 & -0.1886 & -0.1003 & -0.0152 & -0.0613 \\
\hline$-\mathrm{O}(\mathrm{CO}) \mathrm{CH}_{3}$ & 16.039 & -0.1867 & -0.0772 & -0.2131 & -0.0789 & -0.2092 & -0.1170 & 0.0717 & 0.0049 \\
\hline$-\mathrm{P}\left(\mathrm{CH}_{3}\right)_{2}$ & 18.870 & -0.1871 & -0.0756 & -0.2131 & -0.0789 & -0.1908 & -0.1126 & -0.0596 & -0.1421 \\
\hline$-\mathrm{SCH}_{3}$ & 14.155 & -0.1862 & -0.0746 & -0.2131 & -0.0789 & -0.1880 & -0.1073 & 0.0197 & -0.1165 \\
\hline$-\mathrm{C}_{6} \mathrm{H}_{5}$ & 17.047 & -0.1897 & -0.0789 & -0.2131 & -0.0789 & -0.1949 & -0.1183 & -0.0008 & -0.0618 \\
\hline$-\mathrm{CHCH}_{2}$ & 15.313 & -0.1872 & -0.0790 & -0.2131 & -0.0789 & -0.2029 & -0.1147 & 0.0085 & -0.0493 \\
\hline -CCH & 14.296 & -0.1866 & -0.0837 & -0.2131 & -0.0789 & -0.2166 & -0.1286 & 0.0724 & 0.0136 \\
\hline$-\mathrm{CH}_{3}$ & 26.245 & -0.1872 & -0.0724 & -0.2131 & -0.0789 & -0.2070 & -0.1100 & -0.0250 & -0.0447 \\
\hline$-\mathrm{C}\left(\mathrm{CH}_{3}\right)_{3}$ & 28.572 & -0.1859 & -0.0721 & -0.2131 & -0.0789 & -0.2060 & -0.1110 & -0.0299 & -0.0459 \\
\hline$-\mathrm{CH}\left(\mathrm{CH}_{3}\right)_{2}$ & 21.656 & -0.1835 & -0.0742 & -0.2131 & -0.0789 & -0.2064 & -0.1107 & -0.0339 & -0.0476 \\
\hline$-\mathrm{Cl}$ & 14.036 & -0.1874 & -0.0800 & -0.2131 & -0.0789 & -0.2198 & -0.1243 & 0.0841 & 0.0314 \\
\hline$-\mathrm{Br}$ & 11.633 & -0.1873 & -0.0806 & -0.2131 & -0.0789 & -0.2190 & -0.1256 & 0.0832 & 0.0273 \\
\hline$-F$ & 15.924 & -0.1877 & -0.0775 & -0.2131 & -0.0789 & -0.2153 & -0.1167 & 0.0796 & 0.0466 \\
\hline$-(\mathrm{CO}) \mathrm{NHCH}_{3}$ & 13.832 & -0.1858 & -0.0835 & -0.2131 & -0.0789 & -0.2190 & -0.1314 & 0.0318 & 0.0504 \\
\hline$-(\mathrm{CO}) \mathrm{NH}_{2}$ & 17.559 & -0.1861 & -0.0843 & -0.2131 & -0.0789 & -0.2213 & -0.1320 & 0.0381 & 0.0645 \\
\hline$-(\mathrm{CO}) \mathrm{OCH}_{3}$ & 10.102 & -0.1924 & -0.0900 & -0.2131 & -0.0789 & -0.2272 & -0.1362 & 0.0667 & 0.0988 \\
\hline$-(\mathrm{CO}) \mathrm{CH}_{3}$ & 17.874 & -0.1908 & -0.0905 & -0.2131 & -0.0789 & -0.2260 & -0.1356 & 0.0469 & 0.0839 \\
\hline$-(\mathrm{CO}) \mathrm{H}$ & 14.255 & -0.1878 & -0.0944 & -0.2131 & -0.0789 & -0.2301 & -0.1393 & 0.0813 & 0.1192 \\
\hline$-(\mathrm{CO}) \mathrm{F}$ & 11.967 & -0.1882 & -0.0963 & -0.2131 & -0.0789 & -0.2374 & -0.1438 & 0.1017 & 0.1476 \\
\hline$-(\mathrm{CO}) \mathrm{Cl}$ & 9.251 & -0.1881 & -0.1007 & -0.2131 & -0.0789 & -0.2415 & -0.1478 & 0.1217 & 0.1775 \\
\hline$-\mathrm{CF}_{3}$ & 18.900 & -0.1872 & -0.0834 & -0.2131 & -0.0789 & -0.2282 & -0.1345 & 0.0416 & 0.0634 \\
\hline$-\mathrm{CCl}_{3}$ & 12.881 & -0.1874 & -0.0851 & -0.2131 & -0.0789 & -0.2281 & -0.1354 & 0.0571 & 0.0703 \\
\hline$-\mathrm{CN}$ & 12.562 & -0.1887 & -0.0928 & -0.2131 & -0.0789 & -0.2312 & -0.1431 & 0.1562 & 0.1361 \\
\hline$-\mathrm{SOOCH}_{3}$ & 13.994 & -0.1881 & -0.0873 & -0.2131 & -0.0789 & -0.2367 & -0.1419 & 0.0882 & 0.1191 \\
\hline$-\mathrm{NO}_{2}$ & 9.697 & -0.1906 & -0.1104 & -0.2131 & -0.0789 & -0.2435 & -0.1513 & 0.1604 & 0.2136 \\
\hline$-\mathrm{SOOCF}_{3}$ & 10.044 & -0.1892 & -0.0926 & -0.2131 & -0.0789 & -0.2469 & -0.1496 & 0.1322 & 0.1692 \\
\hline -P-PhCl & 14.809 & -0.1899 & -0.0803 & -0.2131 & -0.0789 & -0.1966 & -0.1211 & 0.0120 & -0.0511 \\
\hline -o-PhCl & 14.942 & -0.1866 & -0.0800 & -0.2131 & -0.0789 & -0.2036 & -0.1237 & -0.0115 & -0.0442 \\
\hline$-\mathrm{m}-\mathrm{PhCl}$ & 15.285 & -0.1894 & -0.0805 & -0.2131 & -0.0789 & -0.2006 & -0.1227 & 0.0198 & -0.0374 \\
\hline$-\mathrm{o}-\mathrm{PhCF}_{3}$ & 16.032 & -0.1863 & -0.0805 & -0.2131 & -0.0789 & -0.2064 & -0.1250 & 0.0027 & -0.0446 \\
\hline$-\mathrm{m}-\mathrm{PhCF}_{3}$ & 15.394 & -0.1911 & -0.0814 & -0.2131 & -0.0789 & -0.2010 & -0.1228 & 0.0203 & -0.0342 \\
\hline$-\mathrm{p}-\mathrm{PhCF}_{3}$ & 15.534 & -0.1901 & -0.0819 & -0.2131 & -0.0789 & -0.2027 & -0.1250 & 0.0241 & -0.0298 \\
\hline -p- $\mathrm{PhCH}_{3}$ & 16.231 & -0.1896 & -0.0779 & -0.2131 & -0.0789 & -0.1899 & -0.1160 & -0.0071 & -0.0743 \\
\hline -p-PhOCH${ }_{3}$ & 14.886 & -0.1896 & -0.0770 & -0.2131 & -0.0789 & -0.1821 & -0.1127 & -0.0169 & -0.0984 \\
\hline -op- $\mathrm{Ph}\left(\mathrm{CH}_{3}\right)_{3}$ & 17.184 & -0.1908 & -0.0784 & -0.2131 & -0.0789 & -0.1957 & -0.1164 & -0.0421 & -0.0516 \\
\hline -op- $\mathrm{Ph}\left(\mathrm{OCH}_{3}\right)_{3}$ & 14.443 & -0.1854 & -0.0733 & -0.2131 & -0.0789 & -0.1815 & -0.1118 & -0.0411 & -0.1188 \\
\hline$-\mathrm{NC}_{5} \mathrm{H}_{10}$ & 14.012 & -0.1878 & -0.0622 & -0.2131 & -0.0789 & -0.1552 & -0.0768 & -0.0820 & -0.2018 \\
\hline$-\mathrm{P}(\mathrm{Ph})_{2}$ & 16.281 & -0.1865 & -0.0771 & -0.2131 & -0.0789 & -0.1952 & -0.1163 & -0.0404 & -0.1357 \\
\hline$-\mathrm{N}(\mathrm{Ph})_{2}$ & 8.165 & -0.1859 & -0.0693 & -0.2131 & -0.0789 & -0.1660 & -0.0954 & 0.0078 & -0.1494 \\
\hline
\end{tabular}


Table S4: Dataset for the di-substituted $\mathrm{CoCp}_{2}^{+} \mathrm{OH}^{-}$; B3LYP-D3/(m6-31G*,6-31G**), aqueous PCM.

\begin{tabular}{|c|c|c|c|c|c|c|c|c|c|}
\hline $\begin{array}{c}\text { substituent } \\
\text { Y }\end{array}$ & $\begin{array}{l}\mathrm{BDE} \\
{\left[\frac{\mathrm{kcal}}{\mathrm{mol}}\right]}\end{array}$ & $\begin{array}{c}\mathrm{E}_{\mathrm{HOMO}}^{(1)} \\
{\left[\mathrm{E}_{h}\right]}\end{array}$ & $\begin{array}{c}\mathrm{E}_{\mathrm{LUMO}}^{(1)} \\
{\left[\mathrm{E}_{h}\right]}\end{array}$ & $\begin{array}{c}\mathrm{E}_{\mathrm{HOMO}}^{(2)} \\
{\left[\mathrm{E}_{h}\right]}\end{array}$ & $\begin{array}{c}\mathrm{E}_{\mathrm{LUMO}}^{(2)} \\
{\left[\mathrm{E}_{h}\right]}\end{array}$ & $\begin{array}{c}\mathrm{E}_{\mathrm{HOMO}}^{(3)} \\
{\left[\mathrm{E}_{h}\right]}\end{array}$ & $\begin{array}{c}\mathrm{E}_{\mathrm{LUMO}}^{(3)} \\
{\left[\mathrm{E}_{h}\right]}\end{array}$ & $\begin{array}{c}\mathrm{H}_{C_{6} H_{5}}^{(3)} \\
{[\mathrm{e}]}\end{array}$ & $\begin{array}{c}\mathrm{H}_{C_{5} H_{4}}^{(3)} \\
{[\mathrm{e}]}\end{array}$ \\
\hline$-\mathrm{N}\left(\mathrm{CH}_{3}\right)_{2}$ & 19.101 & -0.1872 & -0.0551 & -0.1787 & -0.0719 & -0.1594 & -0.0809 & 0.0118 & -0.1831 \\
\hline$-\mathrm{OCH}_{3}$ & 18.201 & -0.1865 & -0.0678 & -0.1935 & -0.0727 & -0.1886 & -0.1003 & -0.0152 & -0.0613 \\
\hline$-\mathrm{O}(\mathrm{CO}) \mathrm{CH}_{3}$ & 19.666 & -0.1857 & -0.0805 & -0.2170 & -0.0855 & -0.2092 & -0.1170 & 0.0717 & 0.0049 \\
\hline$-\mathrm{P}\left(\mathrm{CH}_{3}\right)_{2}$ & 20.575 & -0.1894 & -0.0762 & -0.2133 & -0.0814 & -0.1908 & -0.1126 & -0.0596 & -0.1421 \\
\hline$-\mathrm{SCH}_{3}$ & 17.839 & -0.1924 & -0.0745 & -0.2015 & -0.0817 & -0.1880 & -0.1073 & 0.0197 & -0.1165 \\
\hline$-\mathrm{C}_{6} \mathrm{H}_{5}$ & 21.628 & -0.1950 & -0.0815 & -0.1997 & -0.0806 & -0.1949 & -0.1183 & -0.0008 & -0.0618 \\
\hline$-\mathrm{CHCH}_{2}$ & 15.279 & -0.1870 & -0.0828 & -0.2003 & -0.0802 & -0.2029 & -0.1147 & 0.0085 & -0.0493 \\
\hline -CCH & 11.745 & -0.1873 & -0.0909 & -0.2104 & -0.0851 & -0.2166 & -0.1286 & 0.0724 & 0.0136 \\
\hline$-\mathrm{CH}_{3}$ & 30.221 & -0.1870 & -0.0700 & -0.2053 & -0.0778 & -0.2070 & -0.1100 & -0.0250 & -0.0447 \\
\hline$-\mathrm{C}\left(\mathrm{CH}_{3}\right)_{3}$ & 31.220 & -0.1889 & -0.0714 & -0.2040 & -0.0770 & -0.2060 & -0.1110 & -0.0299 & -0.0459 \\
\hline$-\mathrm{CH}\left(\mathrm{CH}_{3}\right)_{2}$ & 37.084 & -0.1861 & -0.0692 & -0.2009 & -0.0945 & -0.2064 & -0.1107 & -0.0339 & -0.0476 \\
\hline$-\mathrm{H}$ & 26.551 & -0.1856 & -0.0747 & -0.2131 & -0.0789 & -0.2110 & -0.1190 & -0.0506 & -0.0575 \\
\hline$-\mathrm{Cl}$ & 12.223 & -0.1882 & -0.0862 & -0.2136 & -0.0847 & -0.2198 & -0.1243 & 0.0841 & 0.0314 \\
\hline$-\mathrm{Br}$ & 9.134 & -0.1880 & -0.0866 & -0.2151 & -0.0841 & -0.2190 & -0.1256 & 0.0832 & 0.0273 \\
\hline$-F$ & 14.953 & -0.1883 & -0.0814 & -0.2121 & -0.0807 & -0.2153 & -0.1167 & 0.0796 & 0.0466 \\
\hline$-(\mathrm{CO}) \mathrm{NHCH}_{3}$ & 12.715 & -0.1850 & -0.0914 & -0.2192 & -0.0863 & -0.2190 & -0.1314 & 0.0318 & 0.0504 \\
\hline$-(\mathrm{CO}) \mathrm{NH}_{2}$ & 16.035 & -0.1854 & -0.0926 & -0.2209 & -0.0867 & -0.2213 & -0.1320 & 0.0381 & 0.0645 \\
\hline$-(\mathrm{CO}) \mathrm{OCH}_{3}$ & 11.519 & -0.1876 & -0.1000 & -0.2242 & -0.0909 & -0.2272 & -0.1362 & 0.0667 & 0.0988 \\
\hline$-(\mathrm{CO}) \mathrm{CH}_{3}$ & 27.119 & -0.1900 & -0.1014 & -0.2164 & -0.1066 & -0.2260 & -0.1356 & 0.0469 & 0.0839 \\
\hline$-(\mathrm{CO}) \mathrm{H}$ & 9.112 & -0.1876 & -0.1074 & -0.2277 & -0.0920 & -0.2301 & -0.1393 & 0.0813 & 0.1192 \\
\hline$-(\mathrm{CO}) \mathrm{F}$ & 5.082 & -0.1905 & -0.1124 & -0.2322 & -0.0943 & -0.2374 & -0.1438 & 0.1017 & 0.1476 \\
\hline$-\mathrm{CF}_{3}$ & 16.559 & -0.1883 & -0.0924 & -0.2245 & -0.0888 & -0.2282 & -0.1345 & 0.0416 & 0.0634 \\
\hline$-\mathrm{CCl}_{3}$ & 10.285 & -0.1874 & -0.0952 & -0.2246 & -0.0894 & -0.2281 & -0.1354 & 0.0571 & 0.0703 \\
\hline$-\mathrm{CN}$ & 4.427 & -0.1913 & -0.1093 & -0.2297 & -0.0944 & -0.2312 & -0.1431 & 0.1562 & 0.1361 \\
\hline$-\mathrm{SOOCH}_{3}$ & 9.324 & -0.1990 & -0.0986 & -0.2313 & -0.0939 & -0.2367 & -0.1419 & 0.0882 & 0.1191 \\
\hline -P-PhCl & 28.664 & -0.1962 & -0.0822 & -0.1991 & -0.0989 & -0.1966 & -0.1211 & 0.0120 & -0.0511 \\
\hline$-\mathrm{o}-\mathrm{PhCl}$ & 16.478 & -0.1905 & -0.0852 & -0.2078 & -0.0797 & -0.2036 & -0.1237 & -0.0115 & -0.0442 \\
\hline$-\mathrm{m}-\mathrm{PhCl}$ & 14.775 & -0.1905 & -0.0857 & -0.2045 & -0.0829 & -0.2006 & -0.1227 & 0.0198 & -0.0374 \\
\hline$-\mathrm{o}-\mathrm{PhCF}_{3}$ & 15.827 & -0.1900 & -0.0864 & -0.2042 & -0.0759 & -0.2064 & -0.1250 & 0.0027 & -0.0446 \\
\hline$-\mathrm{m}-\mathrm{PhCF}_{3}$ & 25.532 & -0.1900 & -0.0860 & -0.2019 & -0.0995 & -0.201 & -0.1228 & 0.0203 & -0.0342 \\
\hline$-\mathrm{p}-\mathrm{PhCF}_{3}$ & 29.069 & -0.1961 & -0.0850 & -0.2024 & -0.0999 & -0.2027 & -0.1250 & 0.0241 & -0.0298 \\
\hline -p- $\mathrm{PhCH}_{3}$ & 31.037 & -0.1920 & -0.0784 & -0.1946 & -0.0972 & -0.1899 & -0.1160 & -0.0071 & -0.0743 \\
\hline -p- $\mathrm{PhOCH}_{3}$ & 30.333 & -0.1922 & -0.0768 & -0.1896 & -0.0966 & -0.1821 & -0.1127 & -0.0169 & -0.0984 \\
\hline -op- $\mathrm{Ph}\left(\mathrm{CH}_{3}\right)_{3}$ & 15.076 & -0.1909 & -0.0834 & -0.2060 & -0.0794 & -0.1957 & -0.1164 & -0.0421 & -0.0516 \\
\hline -op- $\mathrm{Ph}\left(\mathrm{OCH}_{3}\right)_{3}$ & 14.150 & -0.1885 & -0.0734 & -0.1956 & -0.0633 & -0.1815 & -0.1118 & -0.0411 & -0.1188 \\
\hline$-\mathrm{NC}_{5} \mathrm{H}_{10}$ & 20.783 & -0.1882 & -0.0532 & -0.1716 & -0.0652 & -0.1552 & -0.0768 & -0.0820 & -0.2018 \\
\hline$-\mathrm{P}(\mathrm{Ph})_{2}$ & 20.060 & -0.1917 & -0.0799 & -0.2097 & -0.0823 & -0.1952 & -0.1163 & -0.0404 & -0.1357 \\
\hline$-\mathrm{N}(\mathrm{Ph})_{2}$ & 13.750 & -0.1865 & -0.0636 & -0.1866 & -0.0812 & -0.1660 & -0.0954 & 0.0078 & -0.1494 \\
\hline
\end{tabular}


Table S5: Dataset for the hetero-substituted $\mathrm{CoCp}_{2}^{+} \mathrm{OH}^{-}$; B3LYP-D3/(m6-31G*,6-31G**), aqueous PCM.

\begin{tabular}{|c|c|c|c|c|c|c|c|c|c|}
\hline $\begin{array}{c}\text { substituent } \\
\mathrm{Y}\end{array}$ & $\begin{array}{l}\mathrm{BDE} \\
{\left[\frac{k c a l}{m o l}\right]}\end{array}$ & $\begin{array}{c}\mathrm{E}_{\mathrm{HOMO}}^{(1)} \\
{\left[\mathrm{E}_{h}\right]}\end{array}$ & $\begin{array}{c}\mathrm{E}_{\mathrm{LUMO}}^{(1)} \\
{\left[\mathrm{E}_{h}\right]}\end{array}$ & $\begin{array}{c}\mathrm{E}_{\mathrm{HOMO}}^{(2)} \\
{\left[\mathrm{E}_{h}\right]}\end{array}$ & $\begin{array}{c}\mathrm{E}_{\mathrm{LUMO}}^{(2)} \\
{\left[\mathrm{E}_{h}\right]}\end{array}$ & $\begin{array}{c}\mathrm{E}_{\mathrm{HOMO}}^{(3)} \\
{\left[\mathrm{E}_{h}\right]}\end{array}$ & $\begin{array}{c}\mathrm{E}_{\mathrm{LUMO}}^{(3)} \\
{\left[\mathrm{E}_{h}\right]}\end{array}$ & $\begin{array}{c}\mathrm{H}_{C_{6} H_{5}}^{(3)} \\
{[\mathrm{e}]}\end{array}$ & $\begin{array}{c}\mathrm{H}_{C_{5} H_{4}}^{(3)} \\
{[\mathrm{e}]}\end{array}$ \\
\hline$-\mathrm{N}\left(\mathrm{CH}_{3}\right)_{2}$ & 18.871 & -0.1863 & -0.0604 & -0.2053 & -0.0778 & -0.1594 & -0.0809 & 0.0118 & -0.1831 \\
\hline$-\mathrm{OCH}_{3}$ & 18.865 & -0.1858 & -0.0680 & -0.2053 & -0.0778 & -0.1886 & -0.1003 & -0.0152 & -0.0613 \\
\hline$-\mathrm{O}(\mathrm{CO}) \mathrm{CH}_{3}$ & 20.181 & -0.1874 & -0.0755 & -0.2053 & -0.0778 & -0.2092 & -0.1170 & 0.0717 & 0.0049 \\
\hline$-\mathrm{P}\left(\mathrm{CH}_{3}\right)_{2}$ & 23.916 & -0.1859 & -0.0720 & -0.2053 & -0.0778 & -0.1908 & -0.1126 & -0.0596 & -0.1421 \\
\hline$-\mathrm{SCH}_{3}$ & 17.871 & -0.1858 & -0.0720 & -0.2053 & -0.0778 & -0.1880 & -0.1073 & 0.0197 & -0.1165 \\
\hline$-\mathrm{C}_{6} \mathrm{H}_{5}$ & 21.050 & -0.1898 & -0.0761 & -0.2053 & -0.0778 & -0.1949 & -0.1183 & -0.0008 & -0.0618 \\
\hline$-\mathrm{CHCH}_{2}$ & 19.486 & -0.1872 & -0.0760 & -0.2053 & -0.0778 & -0.2029 & -0.1147 & 0.0085 & -0.0493 \\
\hline$-\mathrm{CCH}$ & 18.896 & -0.1874 & -0.0822 & -0.2053 & -0.0778 & -0.2166 & -0.1286 & 0.0724 & 0.0136 \\
\hline$-\mathrm{C}\left(\mathrm{CH}_{3}\right)_{3}$ & 28.871 & -0.1856 & -0.0691 & -0.2040 & -0.0770 & -0.2070 & -0.1100 & -0.0250 & -0.0447 \\
\hline$-\mathrm{CH}\left(\mathrm{CH}_{3}\right)_{2}$ & 27.455 & -0.1863 & -0.0690 & -0.2053 & -0.0778 & -0.2064 & -0.1107 & -0.0339 & -0.0476 \\
\hline$-\mathrm{Cl}$ & 18.382 & -0.1870 & -0.0771 & -0.2053 & -0.0778 & -0.2198 & -0.1243 & 0.0841 & 0.0314 \\
\hline$-\mathrm{Br}$ & 15.831 & -0.1873 & -0.0777 & -0.2053 & -0.0778 & -0.2190 & -0.1256 & 0.0832 & 0.0273 \\
\hline$-\mathrm{F}$ & 20.163 & -0.1871 & -0.0746 & -0.2053 & -0.0778 & -0.2153 & -0.1167 & 0.0796 & 0.0466 \\
\hline$-(\mathrm{CO}) \mathrm{NHCH}_{3}$ & 18.226 & -0.1855 & -0.0805 & -0.2053 & -0.0778 & -0.2190 & -0.1314 & 0.0318 & 0.0504 \\
\hline$-(\mathrm{CO}) \mathrm{NH}_{2}$ & 21.948 & -0.1857 & -0.0809 & -0.2053 & -0.0778 & -0.2213 & -0.1320 & 0.0381 & 0.0645 \\
\hline$-(\mathrm{CO}) \mathrm{OCH}_{3}$ & 14.448 & -0.1914 & -0.0869 & -0.2053 & -0.0778 & -0.2272 & -0.1362 & 0.0667 & 0.0988 \\
\hline$-(\mathrm{CO}) \mathrm{CH}_{3}$ & 22.283 & -0.1904 & -0.0879 & -0.2053 & -0.0778 & -0.2260 & -0.1356 & 0.0469 & 0.0839 \\
\hline$-(\mathrm{CO}) \mathrm{H}$ & 18.769 & -0.1875 & -0.0922 & -0.2053 & -0.0778 & -0.2301 & -0.1393 & 0.0813 & 0.1192 \\
\hline$-(\mathrm{CO}) \mathrm{F}$ & 16.723 & -0.1879 & -0.0934 & -0.2053 & -0.0778 & -0.2374 & -0.1438 & 0.1017 & 0.1476 \\
\hline$-(\mathrm{CO}) \mathrm{Cl}$ & 14.025 & -0.1879 & -0.0981 & -0.2053 & -0.0778 & -0.2415 & -0.1478 & 0.1217 & 0.1775 \\
\hline$-\mathrm{CF}_{3}$ & 23.492 & -0.1870 & -0.0801 & -0.2053 & -0.0778 & -0.2282 & -0.1345 & 0.0416 & 0.0634 \\
\hline$-\mathrm{CCl}_{3}$ & 17.338 & -0.1887 & -0.0833 & -0.2053 & -0.0778 & -0.2281 & -0.1354 & 0.0571 & 0.0703 \\
\hline$-\mathrm{CBr}_{3}$ & 7.366 & -0.1863 & -0.0841 & -0.2053 & -0.0778 & -0.2267 & -0.1357 & 0.0625 & 0.0713 \\
\hline$-\mathrm{CN}$ & 17.359 & -0.1894 & -0.0904 & -0.2053 & -0.0778 & -0.2312 & -0.1431 & 0.1562 & 0.1361 \\
\hline$-\mathrm{SOOCH}_{3}$ & 18.473 & -0.1877 & -0.0844 & -0.2053 & -0.0778 & -0.2367 & -0.1419 & 0.0882 & 0.1191 \\
\hline$-\mathrm{NO}_{2}$ & 14.442 & -0.1900 & -0.1086 & -0.2053 & -0.0778 & -0.2435 & -0.1513 & 0.1604 & 0.2136 \\
\hline$-\mathrm{SOOCF}_{3}$ & 14.559 & -0.1885 & -0.0899 & -0.2053 & -0.0778 & -0.2469 & -0.1496 & 0.1322 & 0.1692 \\
\hline -P-PhCl & 19.765 & -0.1931 & -0.0786 & -0.2053 & -0.0778 & -0.1966 & -0.1211 & 0.0120 & -0.0511 \\
\hline$-\mathrm{O}-\mathrm{PhCl}$ & 19.400 & -0.1867 & -0.0765 & -0.2053 & -0.0778 & -0.2036 & -0.1237 & -0.0115 & -0.0442 \\
\hline$-\mathrm{m}-\mathrm{PhCl}$ & 18.310 & -0.1900 & -0.0788 & -0.2053 & -0.0778 & -0.2006 & -0.1227 & 0.0198 & -0.0374 \\
\hline$-\mathrm{o}-\mathrm{PhCF}_{3}$ & 21.528 & -0.1910 & -0.0804 & -0.2053 & -0.0778 & -0.2064 & -0.1250 & 0.0027 & -0.0446 \\
\hline$-\mathrm{m}-\mathrm{PhCF}_{3}$ & 18.993 & -0.1907 & -0.0788 & -0.2053 & -0.0778 & -0.2010 & -0.1228 & 0.0203 & -0.0342 \\
\hline$-\mathrm{p}-\mathrm{PhCF}_{3}$ & 19.598 & -0.1903 & -0.0792 & -0.2053 & -0.0778 & -0.2027 & -0.1250 & 0.0241 & -0.0298 \\
\hline -p- $\mathrm{PhCH}_{3}$ & 20.240 & -0.1899 & -0.0751 & -0.2053 & -0.0778 & -0.1899 & -0.1160 & -0.0071 & -0.0743 \\
\hline -p-PhOCH 3 & 18.944 & -0.1897 & -0.0741 & -0.2053 & -0.0778 & -0.1821 & -0.1127 & -0.0169 & -0.0984 \\
\hline -op- $\mathrm{Ph}\left(\mathrm{CH}_{3}\right)_{3}$ & 21.387 & -0.1906 & -0.0758 & -0.2053 & -0.0778 & -0.1957 & -0.1164 & -0.0421 & -0.0516 \\
\hline -op- $\mathrm{Ph}\left(\mathrm{OCH}_{3}\right)_{3}$ & 18.668 & -0.1856 & -0.0706 & -0.2053 & -0.0778 & -0.1815 & -0.1118 & -0.0411 & -0.1188 \\
\hline$-\mathrm{NC}_{5} \mathrm{H}_{10}$ & 17.550 & -0.1879 & -0.0604 & -0.2053 & -0.0778 & -0.1552 & -0.0768 & -0.0820 & -0.2018 \\
\hline$-\mathrm{P}(\mathrm{Ph})_{2}$ & 20.692 & -0.1886 & -0.0759 & -0.2053 & -0.0778 & -0.1952 & -0.1163 & -0.0404 & -0.1357 \\
\hline$-\mathrm{N}(\mathrm{Ph})_{2}$ & 11.874 & -0.1852 & -0.0672 & -0.2053 & -0.0778 & -0.1660 & -0.0954 & 0.0078 & -0.1494 \\
\hline
\end{tabular}


Table S6: The derivatives of $\mathrm{CoCp}_{2}^{+} \mathrm{OH}^{-}$characterized by the Bond Dissociation Energies (BDE) values below $3 \mathrm{~kJ} / \mathrm{mol}$, which are excluded from the LSF and FNN analysis. Method: B3LYP-D3/(m6-31G*,6-31G $\left.{ }^{* *}\right)$ within PCM. Note that there are no duplicates within the remaining of data set.

\begin{tabular}{|l|c|c|}
\hline \hline type & substituent & BDE [kcal/mol] \\
\hline mono- & $\mathrm{NO}$ & -2.459 \\
& $\mathrm{CBr}$ & 3.023 \\
\hline di- & $\mathrm{NO}$ & -13.690 \\
& $\mathrm{COCl}$ & 1.685 \\
& $\mathrm{CBr}_{3}$ & 0.873 \\
& $\mathrm{NO}_{2}$ & 0.650 \\
& $\mathrm{SOOCF}_{3}$ & 0.478 \\
\hline hetero- & $\mathrm{NO}$ & 2.431 \\
\hline
\end{tabular}


Table S7: Dataset for the di-substituted $\mathrm{RhCp}_{2}^{+} \mathrm{OH}^{-}$; B3LYP-D3/(m6-31G*,6-31G**), aqueous PCM.

\begin{tabular}{|c|c|c|c|c|}
\hline $\begin{array}{c}\text { substituent } \\
\text { Y }\end{array}$ & $\begin{array}{c}\mathrm{BDE} \\
{[\mathrm{kcal} / \mathrm{mol}]}\end{array}$ & $\begin{array}{c}\mathrm{E}_{\mathrm{HOMO}}^{(1)} \\
{\left[\mathrm{E}_{h}\right]}\end{array}$ & $\begin{array}{c}\mathrm{E}_{\mathrm{LUMO}}^{(1)} \\
{\left[\mathrm{E}_{h}\right]}\end{array}$ & $\begin{array}{c}\mathrm{H}_{C_{6} H_{5}}^{(3)} \\
{[\mathrm{e}]}\end{array}$ \\
\hline$-\mathrm{N}\left(\mathrm{CH}_{3}\right)_{2}$ & 41.811 & -0.2003 & -0.0639 & 0.0118 \\
\hline$-\mathrm{OCH}_{3}$ & 41.604 & -0.2105 & -0.0811 & -0.0152 \\
\hline$-\mathrm{O}(\mathrm{CO}) \mathrm{CH}_{3}$ & 43.128 & -0.2093 & -0.0921 & 0.0717 \\
\hline$-\mathrm{P}\left(\mathrm{CH}_{3}\right)_{2}$ & 48.574 & -0.2110 & -0.0818 & -0.0596 \\
\hline$-\mathrm{SCH}_{3}$ & 41.494 & -0.2121 & -0.0828 & 0.0197 \\
\hline$-\mathrm{C}_{6} \mathrm{H}_{5}$ & 44.571 & -0.2128 & -0.0886 & -0.0008 \\
\hline$-\mathrm{CHCH}_{2}$ & 40.226 & -0.2105 & -0.0906 & 0.0085 \\
\hline -CCH & 40.408 & -0.2105 & -0.1009 & 0.0724 \\
\hline$-\mathrm{CH}_{3}$ & 48.024 & -0.2106 & -0.0764 & -0.0250 \\
\hline$-\mathrm{C}\left(\mathrm{CH}_{3}\right)_{3}$ & 53.461 & -0.2113 & -0.0750 & -0.0299 \\
\hline$-\mathrm{CH}\left(\mathrm{CH}_{3}\right)_{2}$ & 50.535 & -0.2101 & -0.0755 & -0.0339 \\
\hline$-\mathrm{H}$ & 51.042 & -0.2098 & -0.0825 & -0.0506 \\
\hline$-\mathrm{Cl}$ & 36.853 & -0.2115 & -0.0947 & 0.0841 \\
\hline$-\mathrm{Br}$ & 38.798 & -0.2109 & -0.0929 & 0.0832 \\
\hline$-\mathrm{F}$ & 34.460 & -0.2107 & -0.0986 & 0.0796 \\
\hline$-(\mathrm{CO}) \mathrm{NHCH}_{3}$ & 35.390 & -0.2113 & -0.1027 & 0.0318 \\
\hline$-(\mathrm{CO}) \mathrm{NH}_{2}$ & 37.967 & -0.2100 & -0.1057 & 0.0381 \\
\hline$-(\mathrm{CO}) \mathrm{OCH}_{3}$ & 35.331 & -0.2114 & -0.1152 & 0.0667 \\
\hline$-(\mathrm{CO}) \mathrm{CH}_{3}$ & 34.888 & -0.2122 & -0.1159 & 0.0469 \\
\hline$-(\mathrm{CO}) \mathrm{H}$ & 28.736 & -0.2109 & -0.1247 & 0.0813 \\
\hline$-(\mathrm{CO}) \mathrm{F}$ & 23.271 & -0.2159 & -0.1312 & 0.1017 \\
\hline$-\mathrm{CF}_{3}$ & 35.078 & -0.2138 & -0.1065 & 0.0416 \\
\hline$-\mathrm{CCl}_{3}$ & 37.865 & -0.2121 & -0.1065 & 0.0571 \\
\hline$-\mathrm{CN}$ & 31.227 & -0.2160 & -0.1143 & 0.1562 \\
\hline$-\mathrm{SOOCH}_{3}$ & 36.486 & -0.2205 & -0.1083 & 0.0882 \\
\hline -P-PhCl & 44.795 & -0.2122 & -0.0912 & 0.0120 \\
\hline$-\mathrm{O}-\mathrm{PhCl}$ & 44.579 & -0.2122 & -0.0927 & -0.0115 \\
\hline$-\mathrm{m}-\mathrm{PhCl}$ & 44.713 & -0.2124 & -0.0923 & 0.0198 \\
\hline$-\mathrm{o}-\mathrm{PhCF}_{3}$ & 44.265 & -0.2122 & -0.0969 & 0.0027 \\
\hline$-\mathrm{m}-\mathrm{PhCF}_{3}$ & 41.839 & -0.2134 & -0.0949 & 0.0203 \\
\hline$-\mathrm{p}-\mathrm{PhCF}_{3}$ & 41.416 & -0.2127 & -0.0997 & 0.0241 \\
\hline -p-PhCH ${ }_{3}$ & 44.970 & -0.2122 & -0.0870 & -0.0071 \\
\hline -p- $\mathrm{PhOCH}_{3}$ & 44.501 & -0.2100 & -0.0870 & -0.0169 \\
\hline -op- $\mathrm{Ph}\left(\mathrm{CH}_{3}\right)_{3}$ & 48.239 & -0.2128 & -0.0866 & -0.0421 \\
\hline -op- $\mathrm{Ph}\left(\mathrm{OCH}_{3}\right)_{3}$ & 46.985 & -0.2110 & -0.0833 & -0.0411 \\
\hline$-\mathrm{NC}_{5} \mathrm{H}_{10}$ & 58.578 & -0.1947 & -0.0615 & -0.0820 \\
\hline$-\mathrm{P}(\mathrm{Ph})_{2}$ & 55.377 & -0.2118 & -0.0834 & -0.0404 \\
\hline$-\mathrm{N}(\mathrm{Ph})_{2}$ & 41.774 & -0.1969 & -0.0716 & 0.0078 \\
\hline
\end{tabular}


Table S8: The optimal coefficients of the LSF models using the Hirshfeld charge on $\mathrm{C}_{6} \mathrm{H}_{5}$.

\begin{tabular}{cccccc}
\hline \hline coefficient & $\mathrm{LSF}_{1}$ & $\mathrm{LSF}_{1}^{\prime}$ & $\mathrm{LSF}_{2}$ & $\mathrm{LSF}_{2}^{\prime}$ & $\mathrm{LSF}_{3}$ \\
\hline $\mathrm{c}_{0}$ & -68.223 & -55.220 & 0.495 & 6.101 & -74.413 \\
$\mathrm{c}_{1}$ & -0.855 & -0.717 & 0.378 & 0.544 & -0.551 \\
$\mathrm{c}_{2}$ & 0.074 & 0.191 & 0.483 & -0.954 & 0.613 \\
$\mathrm{c}_{3}$ & 0.242 & -73.947 & -0.644 & -0.578 & 0.319 \\
$\mathrm{c}_{4}$ & 0.072 & - & -0.855 & 0.527 & 0.770 \\
$\mathrm{c}_{5}$ & - & - & -0.624 & -42.651 & -0.869 \\
$\mathrm{c}_{6}$ & - & - & -0.159 & -75.462 & -1.771 \\
$\mathrm{c}_{7}$ & - & - & 0.450 & - & -0.645 \\
$\mathrm{c}_{8}$ & - & - & 0.467 & - & 0.104 \\
$\mathrm{c}_{9}$ & - & - & - & - & 0.284 \\
$\mathrm{c}_{10}$ & - & - & - & - & -0.020 \\
$\mathrm{k}^{2}$ & 100.751 & - & 8.980 & - & 437.979 \\
$\mathrm{H}_{0}^{2}$ & 0.076 & - & 0.077 & - & 0.084 \\
$\mathrm{k}^{3}$ & - & - & 31.809 & - & 39.667 \\
$\mathrm{H}_{0}^{3}$ & - & - & 0.028 & - & 0.018 \\
\hline \hline
\end{tabular}

Table S9: The optimal coefficients of the LSF models using the Hirshfeld charge on $\mathrm{C}_{5} \mathrm{H}_{4}$.

\begin{tabular}{cccccc}
\hline \hline coefficient & $\mathrm{LSF}_{1}$ & $\mathrm{LSF}_{1}^{\prime}$ & $\mathrm{LSF}_{2}$ & $\mathrm{LSF}_{2}^{\prime}$ & $\mathrm{LSF}_{3}$ \\
\hline $\mathrm{c}_{0}$ & -125.018 & -63.211 & -17.155 & -58.725 & -32.743 \\
$\mathrm{c}_{1}$ & -1.367 & -1.005 & 0.278 & 0.380 & -0.195 \\
$\mathrm{c}_{2}$ & 26.002 & 0.714 & 14686.282 & -0.897 & 0.837 \\
$\mathrm{c}_{3}$ & 0.340 & 17.139 & -0.735 & -0.893 & 0.276 \\
$\mathrm{c}_{4}$ & -43.289 & - & -29936.915 & 0.508 & 0.628 \\
$\mathrm{c}_{5}$ & - & - & -0.703 & -36.876 & -0.854 \\
$\mathrm{c}_{6}$ & - & - & 0.079 & -79.615 & -1.483 \\
$\mathrm{c}_{7}$ & - & - & 0.690 & - & -0.615 \\
$\mathrm{c}_{8}$ & - & - & -0.026 & - & 0.282 \\
$\mathrm{c}_{9}$ & - & - & - & - & 0.184 \\
$\mathrm{c}_{10}$ & - & - & - & - & -0.379 \\
$\mathrm{k}^{2}$ & 70.136 & - & 12.135 & - & 123.999 \\
$\mathrm{H}_{0}^{2}$ & 0.171 & - & 0.990 & - & 0.087 \\
$\mathrm{k}^{3}$ & - & - & 253.438 & - & 270.548 \\
$\mathrm{H}_{0}^{3}$ & - & - & 0.003 & - & 0.005 \\
\hline \hline
\end{tabular}

Table S10: Hyperparameters of the FNN models.

\begin{tabular}{ccccccc}
\hline \hline model & $H$-charge & nodes & learning rate & weight decay & batch size & epoch \\
\hline FNN2 & $\mathrm{C}_{6} \mathrm{H}_{5}$ & 180 & 0.001 & $10^{-4}$ & 16 & 3000 \\
& $\mathrm{C}_{5} \mathrm{H}_{4}$ & 120 & 0.001 & $10^{-8}$ & 16 & 3000 \\
\hline \multirow{2}{*}{ FNN3 } & $\mathrm{C}_{6} \mathrm{H}_{5}$ & 120 & 0.001 & $10^{-4}$ & 16 & 3000 \\
& $\mathrm{C}_{5} \mathrm{H}_{4}$ & 100 & 0.001 & $10^{-8}$ & 16 & 3000 \\
\hline \hline
\end{tabular}




\section{References}

(1) Zhu, T.; Xu, S.; Rahman, A.; Dogdibegovic, E.; Yang, P.; Pageni, P.; Kabir, M. P.; Zhou, X.-d.; Tang, C. Cationic Metallo-Polyelectrolytes for Robust Alkaline AnionExchange Membranes. Angew.Chem. 2018, 57, 2388-2392.

(2) Phung, Q. M.; Vancoillie, S.; Pierloot, K. Theoretical Study of the Dissociation Energy of First-Row Metallocenium Ions. J. Chem. Theory Comput. 2014, 10, 3681-3688. 